\title{
O que resta de marketing para o Chief Marketing Officer?
}

\author{
MEL GiRÃo ${ }^{1}$ \\ ERIK FERNANDES ${ }^{1}$
}

${ }^{1}$ Fundação Getulio Vargas (FGV EAESP) / Escola de Administração de EMpresas de SÃo Paulo, SÃo PaUlo - SP, Brasil

\section{Resumo}

O Chief Marketing Officer (CMO), ${ }^{1}$ ou principal executivo de marketing, tem uma função estratégica nas empresas e está no centro das transformações digitais e de mudanças nos padrões de consumo com as quais lidamos hoje. No entanto, seu turnover é o mais alto entre os executivos da C-suite. Este trabalho procura identificar os aspectos que influenciam esse alto turnover. Para atender ao objetivo da pesquisa, utilizou-se o método da revisão sistemática da literatura. Os resultados mostram que o perfil, a formação e o papel dos CMOs nas empresas, bem como seu relacionamento sobretudo com o CEO, estão no cerne desta questão. O CMO, diante dos desafios da era digital, deve se reinventar, incluindo em sua formação elementos mais qualitativos e analíticos, incorporando novos métodos e ferramentas de análise do comportamento do cliente e do mercado nesse ambiente digital. Além disso, deve preservar e reforçar sua função de representante do cliente na organização por meio de um trabalho articulado de engajamento e coordenação de outros C-executives, muitos deles em funções novas e adjacentes à sua, de modo a dar suporte às decisões estratégicas do CEO e ajudar a empresa a alavancar seu desempenho. De forma ampla, este artigo pretende colaborar para a atualização de acadêmicos e profissionais de marketing em relação às mudanças que têm ocorrido na atuação dos CMOs, ajudando-os a moldar seu escopo no futuro.

Palavras-chave: Chief Marketing Officer. Diretor de marketing. Executivos de marketing. Rotatividade. C-suite.

\section{What is left of marketing for the Chief Marketing Officer?}

\begin{abstract}
The CMO (Chief Marketing Officer) plays a strategic role in a company and is at the heart of digital transformations and changes in the consumption patterns we face today. However, the turnover of this position is the highest among C-suite executives. This work seeks to identify the aspects that influence this high turnover through a systematic literature review. The results show that the profile, educational background, and role of CMOs in companies and their relationship mainly with the CEO are at the heart of this issue. The CMO, facing the challenges of the digital age, must reinvent themselves to include more qualitative and analytical elements in their training, incorporating new methods and tools for analyzing customer and market behavior in this digital environment. They also need to preserve and reinforce their role as the customer's voice representative in the organization through engagement and coordination with other C-executives, many of them in new and adjacent roles, to support strategic decisions, and help the company leverage its performance. This article intends to collaborate to update academics and those in marketing concerning the changes that have occurred in the performance of CMOs and help them shape their scope in the future.
\end{abstract}

Keywords: Chief Marketing Officer. Marketing director. Marketing executives. Turnover. C-suite.

\section{¿Qué le queda de marketing al chief marketing officer?}

\section{Resumen}

El CMO (director de marketing) juega un papel estratégico en las empresas, y está en el centro de las transformaciones digitales y los cambios en los patrones de consumo a los que nos enfrentamos hoy. Sin embargo, su rotación es la más alta entre los ejecutivos de c-suite. Este trabajo busca identificar los aspectos que influyen en esta alta rotación, y para cumplir con el objetivo de la investigación se utilizó el método de revisión sistemática de la literatura. Los resultados muestran que el perfil, la formación y el rol de los CMO en las empresas y su relación principalmente con el CEO están en el centro de esta cuestión. EI CMO, ante los retos de la era digital, debe reinventarse, incluyendo más elementos cualitativos y analíticos en su formación, incorporando nuevos métodos y herramientas para analizar el comportamiento de los clientes y del mercado en este entorno digital. Además, debe preservar y reforzar su papel como representante del cliente en la organización a través de un trabajo articulado de compromiso y coordinación de otros c-ejecutivos, muchos de ellos en roles nuevos y adyacentes al suyo, con el fin de respaldar las decisiones estratégicas del CEO y ayudar a la empresa a apalancar su desempeño. En términos generales, este artículo pretende colaborar para actualizar a académicos y especialistas en marketing en relación con los cambios que se han producido en el desempeño de los CMO y ayudarlos a dar forma a su campo de acción en el futuro.

Palabras clave: Chief marketing officer. Director de marketing. Ejecutivo de mercadeo. Rotación. C-suite.

${ }^{1}$ O grupo desses executivos sêniores das empresas também é conhecido como C-Suite ou C-Executives. CEO é Chief Executive Officer. CIO é Chief Information Officer. CSO é Chief Sales Officer. 


\section{INTRODUÇÃO}

Quando Philip Kotler soou o alarme em 2003, com seu artigo "The decline of Marketing: from four Ps to one P", Chiefs Marketing Officers (CMOs) e diretores executivos da área se debatiam com descrições de cargo muito estreitas, com excessiva ênfase em promoções e propaganda (Malter \& Genesan, 2005). Esse cenário tem causado frustração crescente entre tais profissionais, cuja rotina é permeada por expectativas não factíveis de seus Chiefs Executive Officers (CEOs), assim como suporte organizacional inadequado de suas companhias. Dessa forma, taxas crescentes de turnover têm sido observadas em diversos países. Em 2004, uma pesquisa anual realizada pela empresa de recrutamento de executivos SpencerStuart (Welch, 2004) afirmou que a média de permanência de um CMO nas 100 maiores empresas de bens de consumo dos Estados Unidos era de menos de 24 meses. No mercado de telecomunicações, mostrou o estudo, a situação era ainda mais grave: 15 meses, em média. Esses prazos são um contraste mesmo em relação ao tempo médio de permanência dos CEOs, apontado pelo estudo como sendo de 54 meses, fazendo do $\mathrm{CMO}$ o cargo mais arriscado da $\mathrm{C}$-suite.

Mais tarde, em 2007, o relatório da Consultoria de Negócios McKinsey Quarterly (Court, 2007) publicou dados sobre a alta mortalidade dos CMOs, em 2011, a Forbes (Whitler, 2011) fez nova matéria sobre a situação, mostrando um cenário inalterado. Desde então, a situação parece ter piorado. Uma pesquisa da empresa de recrutamento de executivos RussellReynolds, citada em Gee (2016), mostra que o turnover dos CMOs atingiu seu pico na série histórica, com 48\% das companhias trocando seu executivo sênior de marketing a cada 12 meses. Outro estudo, realizado em 2019 pela também empresa de recrutamento SpencerStuart (2019), afirma que o turnover do CMO, nos mais diversos setores da economia americana, atingiu níveis sem precedentes. Dados compilados em 2017 por Kimberly Whitley (ver Gráfico 1) dão detalhes da situação.

\section{Gráfico 1}

Permanência no cargo: anos versus percentual

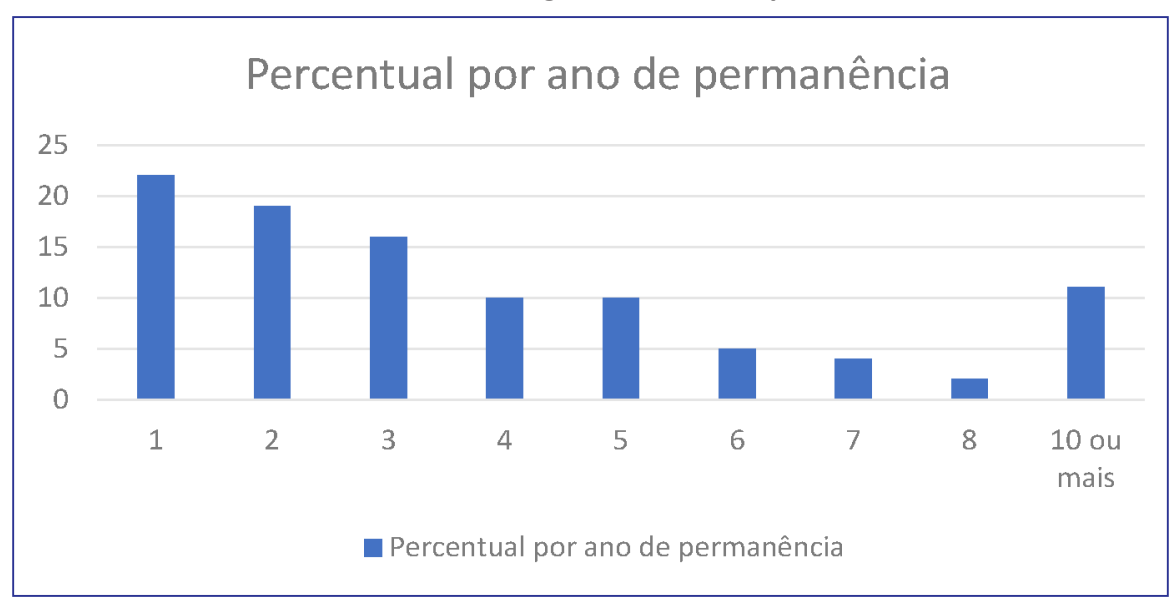

Fonte: Whitler (2014, 2015 como citado em Whitler \& Morgan, 2017, p. 49).

O papel do CMO na organização, no entanto, é estratégico para liderança, governança e resultados das empresas (Germann, Ebbes \& Grewal, 2015; Nath \& Mahajan, 2011; Srinivasan \& Ramani, 2019), e há comprovação de correlação positiva entre o CMO e o crescimento de vendas da empresa (Nath \& Mahajan, 2008, 2011, 2017; Weinzimmer, Bond, Houston \& Nystrom, 2013). Além disso, a presença do CMO na C-Suite traz o consumidor para as decisões centrais das empresas, o que impacta positivamente sua performance (Kerin, 2005; McGovern, Quelch \& Crawford, 2004). Assim, o alto turnover dos CMOs deveria ser evitado, mas, ao que parece, não é o que está ocorrendo.

O resultado é que diversas companhias perdem oportunidades por não perceberem as dificuldades dos CMOs em realizar impactos relevantes nas atividades da organização. De acordo com um estudo da consultoria de negócios Deloitte ( $O^{\prime} B r i e n$, Veenstra \& Murphy, 2018), realizado em 2018, essa perda pode ser financeiramente relevante. Uma análise de portfólio mostrou que ações de companhias que têm um CMO na sua diretoria executiva trouxeram resultados de longo prazo significativamente mais altos aos seus stakeholders. 
Tabela 1

CMO: o cargo mais arriscado da C-Suite

\begin{tabular}{l|c|c|c|c|c|c}
\hline Tempo médio no cargo (anos) & CEO & CFO & CIO & CMO & CHRO & $\begin{array}{c}\text { Média da } \\
\text { C-Suite }\end{array}$ \\
\hline Bens de consumo & 8.0 & 5.1 & 4.5 & $\mathbf{3 . 6}$ & 4.9 & 5.2 \\
\hline Energia & 6.1 & 5.0 & 4.5 & $\mathbf{4 . 6}$ & 5.3 & 5.1 \\
\hline Serviços financeiros & 9.7 & 5.5 & 4.1 & $\mathbf{5 . 1}$ & 5.1 & 5.9 \\
\hline Indústria & 6.7 & 4.9 & 4.0 & $\mathbf{4 . 1}$ & 4.6 & 4.9 \\
\hline Life sciences & 9.4 & 6.0 & 4.1 & $\mathbf{3 . 1}$ & 5.1 & 5.5 \\
\hline Serviços profissionais & 9.2 & 5.0 & 4.5 & $\mathbf{4 . 1}$ & 5.1 & 5.6 \\
\hline Tecnologia & 7.9 & 4.9 & 4.4 & $\mathbf{4 . 3}$ & 5.2 & 5.3 \\
\hline Média geral & $\mathbf{8 . 0}$ & $\mathbf{5 . 1}$ & $\mathbf{4 . 3}$ & $\mathbf{4 . 1}$ & $\mathbf{5 . 0}$ & $\mathbf{5 . 3}$ \\
\hline
\end{tabular}

Fonte: Korn Ferry (2017 como citado em Whitler \& Morgan, 2017, p. 52).

Dessa forma, algumas das preocupações atuais mais latentes dos profissionais de marketing envolvem a dinâmica da mudança constante de seu papel nas organizações, mais especificamente "como liderar as mudanças num cenário em que as próprias metas mudam, como contrabalançar demandas de curto e longo prazos e como todas as mudanças em seu descritivo de cargo afetam a viabilidade das estratégias de marketing" (Morgan, Whitler, Feng \& Chasi, 2019, p. 26).

De fato, muitas companhias já veem a necessidade de o cargo de CMO ter maior amplitude e ser mais estratégico do ponto de vista corporativo, ou seja, menos dedicado a ações puramente táticas (Whitler \& Morgan, 2017). Ainda assim, há uma demanda para que os $\mathrm{CMO}$ s tenham uma atuação estratégica e de alto nível, mas com frequência eles não recebem a alçada e os recursos para serem efetivos (Crosby \& Johnson, 2005; Kerin, 2005; McGovern \& Quelch, 2004; Silver, 2003).

Como Kimberly Whitter comentou na Forbes, em 2011, para várias companhias, perder um CMO muitas vezes parece aceitável. O CMO é, de forma geral, a voz do consumidor dentro das organizações, responsável pela maior parte do desenho da interface desses consumidores com a empresa. Então, por que alguém permitiria, justamente nessa área, instabilidade, inconsistência e confusão geradas pelo alto turnover, sem mencionar os altos custos de recrutamento e reposição de pessoal?

Os estudos sobre as possíveis explicações do turnover do CMO é matéria pouco explorada, se comparados com a literatura disponível sobre turnover de CEO e COO. Essa lacuna é surpreendente, uma vez que o estudo dos C-Executives é crucial para entendermos seu papel no top management team (Nath \& Mahajan, 2017). O objetivo deste trabalho é contribuir para enriquecer essa discussão.

A multiplicidade do escopo da atuação de um profissional de marketing, que não será objeto de discussão deste trabalho, passou de criação de valor (Alderson, 1957), orientação ao mercado (Kohli \& Jaworski, 1990), para construção e gestão de marcas (Fuchs \& Diamantopoulos, 2010), com foco no consumidor (Grönroos, 2006). A questão fundamental em nossa discussão é que tais focos de atuação são, infelizmente, de pouca relevância para membros do conselho de administração e para CEOs, em particular (Fournaise Marketing Group, 2011). Como consequência, é improvável que esses assuntos sejam discutidos pela alta administração das empresas durante o desenvolvimento das estratégias de suas companhias (Wright \& Russell, 2012).

Assim, o marketing, uma vez objeto de desejo de estudantes de pós-graduação e destino de executivos que buscavam crescimento profissional por meio de migrações internas nas Companhias - cortesia da popularização dos conceitos de cadeia de valor e do arcabouço da estratégia competitiva de Porter (Wirtz, Tuzovic \& Kuppelwieser, 2014) -, foi demovido de um papel estratégico para funções táticas ou de apoio nos últimos 30 anos (Klaus, Edvardsson \& Maklan, 2014; Strandvik, Holmlund \& Grönroos, 2014). Pesquisas atribuem essa decadência a 3 razões principais:

1. Má fama ou má reputação atribuída ao marketing (Gummesson, Kuusela \& Närvänen, 2014).

2. Atuação limitada, ligada a atividades funcionais ou operacionais, que se ressentem de uma visão mais ampla e estratégica (Webster \& Lusch, 2013). 
3. Não uso de evidências empíricas mostrando a relação de causa e efeito entre as atividades de marketing, os resultados e a performance das companhias (Verhoef et al., 2011).

Nesse contexto, devemos nos perguntar: quem, dentro das empresas, se torna especialista nos padrões e nos hábitos de compra dos consumidores? Quem será o responsável por atividades como administração de portfólio de marcas, preços, pesquisas de mercado de longo prazo, segmentação de mercado e gestão do relacionamento com o cliente? Em várias empresas, hoje, essas perguntas não têm respostas fáceis (Whitler, Boyd \& Morgan, 2017).

\section{METODOLOGIA}

Com base numa revisão da literatura (Kitchenham, 2004; Petticrew \& Roberts, 2008), procuramos interpretar as pesquisas disponíveis relevantes, indicando gaps e caminhos para futuras pesquisas sobre a evolução da relevância do CMO, suas relações com os outros $C$-Suite executives e os impactos sobre o mandato dessa função nos novos contextos mercadológicos.

Para tanto, valemo-nos da base de dados Ebsco e do Google Scholar. Usamos 4 palavras-chave como fonte primaria da pesquisa: Chief Marketing Officer, C-suite, tenure e turnover. Esses termos, em ambas as bases de dados, foram associadas ao operador booleano AND e OR, nas seguintes configurações: Chief Marketing Officer AND C-suite; Chief Marketing officer AND C-Suite AND tenure OR turnover; Chief Marketing Officer AND tenure OR turnover.

Num primeiro momento, a pesquisa foi limitada ao período de 2004 a 2019, sendo posteriormente expandida de 2014 a 2020. $\mathrm{Na}$ base de dados Ebsco, fizemos 2 rodadas de pesquisa: uma sem filtro de publicação e outra filtrando somente periódicos científicos e revistas acadêmicas. No Google Scholar, pesquisamos artigos cujas palavras constassem no título.

A pesquisa resultou (veja o Quadro 1) em 142 apontamentos na base dados Ebsco para a combinação de Chief Marketing Officer + C-suite, com 15 apontamentos em periódicos e revistas acadêmicas e somente 1 no Google Scholar. A combinação das palavras Chief Marketing Officer + C-suite + tenure ou turnover gerou 9 resultados (com apenas 1 em periódicos ou revistas acadêmicas e sem nenhum apontamento no Google Scholar). Já a combinação Chief Marketing Officer + tenure ou turnover retornou 380 resultados (sendo somente 7 em periódicos ou revistas acadêmicas e 1 apontamento no Google Scholar).

Por fim, selecionamos os artigos que apresentavam análises mais amplas, e não casos específicos de empresas, concentrando-nos em periódicos científicos e revistas acadêmicas. Num segundo momento, acrescentamos alguns artigos anteriores a 2014 que consideramos relevantes para o assunto.

Quadro 1

Resultados da revisão de literatura

\begin{tabular}{|c|c|c|c|c|}
\hline Palavras-chave & $\begin{array}{c}\text { Número de } \\
\text { apontamentos }\end{array}$ & Período & Filtro & $\begin{array}{c}\text { Base de } \\
\text { dados }\end{array}$ \\
\hline Chief Marketing Officer + C Suite & 142 & \multirow{9}{*}{$2004-2020$} & \multirow{3}{*}{$\begin{array}{l}\text { Sem filtro de tipo de } \\
\text { publicação }\end{array}$} & \multirow{6}{*}{ Ebsco } \\
\hline Chief Marketing Officer + C Suite + (Tenure or Turnover) & 9 & & & \\
\hline Chief marketing Officer + (Tenure or Turnover) & 380 & & & \\
\hline Chief Marketing Officer + C Suite & 15 & & \multirow{3}{*}{$\begin{array}{c}\text { Com filtro: } \\
\text { periódicos } \\
\text { científicos, revistas } \\
\text { acadêmicas }\end{array}$} & \\
\hline Chief Marketing Officer + C Suite + (Tenure or Turnover) & 1 & & & \\
\hline Chief marketing Officer + (Tenure or Turnover) & 7 & & & \\
\hline Chief Marketing Officer + C Suite & 1 & & \multirow{3}{*}{$\begin{array}{l}\text { Com filtro: } \\
\text { palavras-chaves } \\
\text { no título }\end{array}$} & \multirow{3}{*}{$\begin{array}{l}\text { Google } \\
\text { Scholar }\end{array}$} \\
\hline Chief Marketing Officer + C Suite + (Tenure or Turnover) & 0 & & & \\
\hline Chief marketing Officer + (Tenure or Turnover) & 1 & & & \\
\hline
\end{tabular}

Fonte: Elaborado pelos autores. 


\section{ALTA VOLATILIDADE: POSSÍVEIS RAZÕES}

A volatilidade dos $\mathrm{CMOs}$ nas empresas pode se fundamentar em vários motivos que se entrelaçam. A aceleração visível da cultura digital tem características sistêmicas que afetam toda a sociedade com base numa rede intricada de inter-relações entre clientes e toda a cadeia de produção, por sua vez alimentada e alimentando interações sociais, tecnológicas e econômicas complexas e dinâmicas (Perez, 2010). O marketing não é imune a tais transformações; ao contrário, é diretamente impactado por elas. Como afirma Nadeem (2015, p. 28, grifo nosso): "Digital não tem sido somente uma mudança no marketing; tem sido a mudança no marketing". Consequentemente, esse cenário também altera os parâmetros base para o exercício função do CMO: o perfil de atuação, o conhecimento ou os insights sobre as necessidades dos clientes (explícitas e latentes), o domínio de ferramentas e conhecimentos necessários para aproveitar oportunidades, a deteç̧ão de ameaças e a geração de vantagens competitivas no mercado, além da gestão e da formação de equipes capazes de enfrentar esses desafios nos novos cenários gerados por mudanças sistêmicas.

Por outro lado, o caráter estatístico e analítico que caracteriza o novo ferramental de ação mercadológica nesse ambiente digital parece exacerbar as dificuldades já conhecidas do CMO de lidar com seus pares da C-Suite - e mesmo seu CEO - com formação em ciências exatas (ou similar) e uma visão mais imediatista (Nath \& Bharadwaj, 2020). A consequência disso é o esvaziamento de muitas responsabilidades para outras funções com facilidade de domínio do ferramental mais analítico. Nesse contexto, os CMOs têm sido criticados por sua inabilidade para apresentar sólidas evidências da efetividade dos grandes gastos financeiros feitos em ações promocionais e construção de marcas (Key \& Keen, 2020; Verhoef \& Leeflang, 2009). Essa percebida falta de accountability gera uma redução na influência das áreas de marketing nas tomadas de decisão estratégicas (Verhoef et al., 2009; Webster, 2005) e provoca um ressentimento nas outras áreas da companhia (Press, 2013).

O fato é que o CMO ideal hoje deve dominar habilidades, do ponto de vista cognitivo, algo conflitantes: de um lado, o que podemos chamar de soft skills, como empatia com consumidores, habilidade de criar, motivar e envolver seus pares e consumidores; de outro, as hard skills, como capacidade de gerir grandes projetos, desenhar estruturas de bases de dados e especificar modelos estatísticos e digitais. Essas habilidades são também chamadas de right (creative) and left (analytical) brain skills, e sua conjugação exige não só um imenso suporte das instituições que formam esses profissionais, mas, sobretudo, um perfil muito distinto e específico.

\section{QUESTÃO DE PERFIL?}

Num artigo de 2017, Whitler e Morgan sugeriram que a maior parte dos cargos de CMOs é desenhada e descrita de forma frágil e superficial, pelo fato de o papel do CMO não estar aderente com a autoridade recebida da companhia nem com responsabilidades ou metas atribuídas ao cargo. Além disso, novos títulos e funções corporativas surgiram recentemente, muitos deles gerados pelo novo contexto digital, o que torna o desenho do perfil CMO ainda mais complexo (Nadeem, 2015).

Whitler e Morgan (2017) argumentam que existem 3 perfis fundamentais de CMOs e que, para uma contratação bem-sucedida, as companhias precisam ter clareza do tipo de profissional de que necessitam:

- O estrategista, que toma decisões sobre o posicionamento de mercado da empresa, criando e desenvolvendo produtos. Ele/ela desenha a estratégia de crescimento da companhia e é responsável por inovação via insights dos consumidores ou clientes com o uso de novas tecnologias. Seriam $31 \%$ da demanda por CMOs.

- O vendedor, que alavanca as vendas por meio de publicidade e promoção, além de conteúdo digital, mídias sociais, relações públicas e eventos, e responderia por $46 \%$ das contratações de CMOs.

- O líder com visão ampla, que tem gestão e responsabilidade pelo P\&L (ou demonstrativo de resultados) e desempenha as duas funções acima. É o responsável pelo desenho da estratégia de crescimento sustentável e supervisiona a estrutura comercial da companhia. Também desenvolve produtos, é responsável pela argumentação de vendas e pelo desenho da distribuição e do preço, além da comunicação de marketing. 
Segundo Whitler e Morgan (2017), a solução para a diminuição do turnover dos CMOs seria uma correta identificação das necessidades das empresas, que permitiria uma contratação mais precisa desses profissionais. Mas, sob a perspectiva de Kotler (2003), os primeiros 2 tipos de perfil - o estrategista e o vendedor - seriam amputados em relação ao profissional de marketing comme il faut. Assim, assumir que diversas empresas precisam de um profissional com menos habilidades do que um profissional em sua plenitude não é uma espécie de profecia autorrealizável? Dito de outra forma: a empresa acha que precisa de "menos", contrata "menos" e acaba insatisfeita?

\section{A FALTA DE SINTONIA ENTRE A FORMAÇÃO ACADÊMICA E O NOVO CENÁRIO DIGITAL}

O'Brien (2016) indica que as atividades de marketing historicamente eram relacionadas com criatividade e uma abordagem intuitiva do mercado. Essa atitude era resultado da formação baseada fundamentalmente em ciências sociais com fortes vieses em propaganda, onde uma postura mais subjetiva era preponderante.

Somente no início da década de 2000 é que precursores como Kotler (2003) apontaram a necessidade de o CMO desenvolver um conhecimento mais aprofundado em análise e linguagem financeira, além de novas tecnologias.

\section{Gráfico 2}

Principais fatores que direcionam o sucesso do CMO

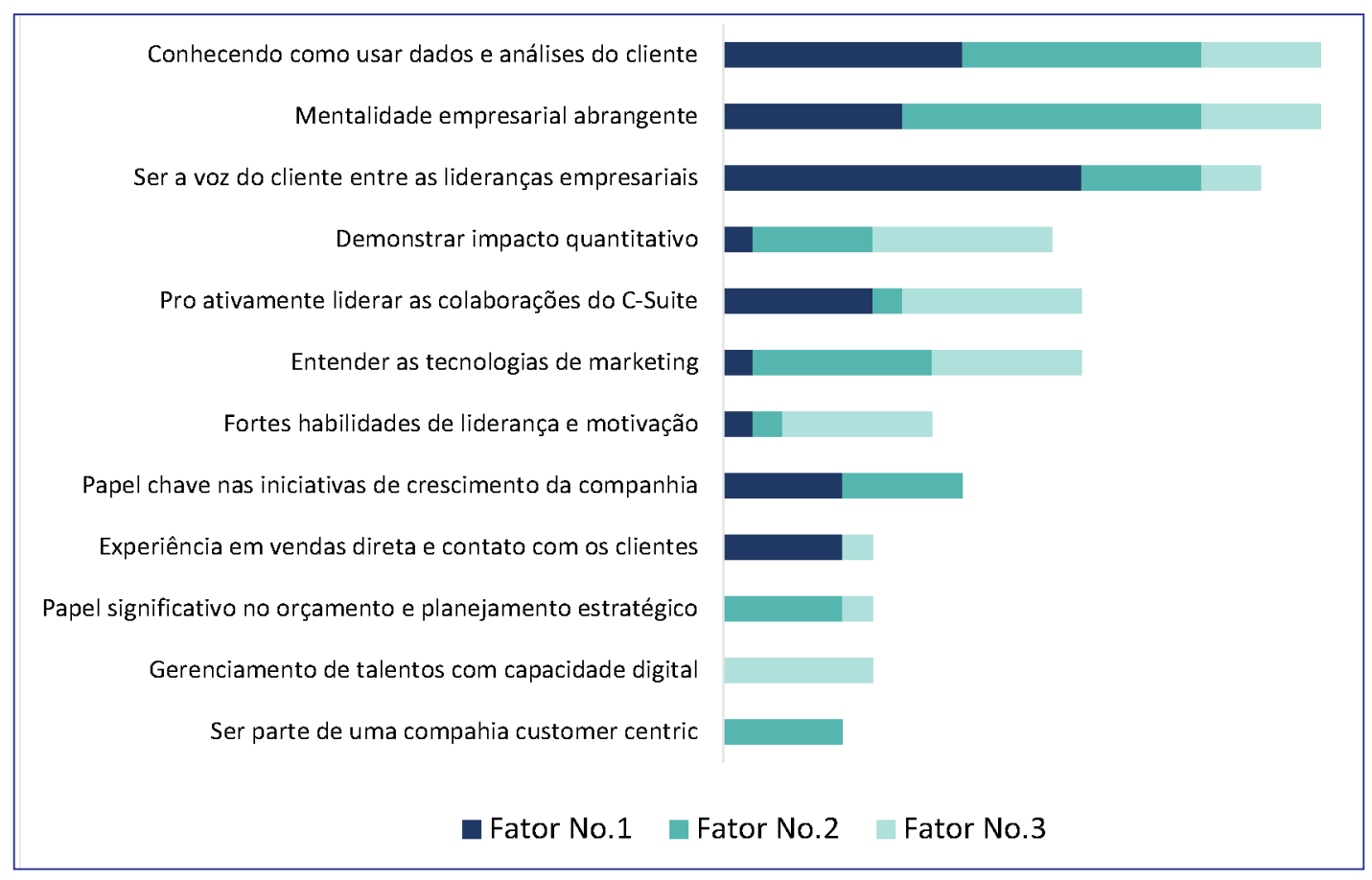

Fonte: O’Brien et al. (2018).

A partir dessa data, os meios digitais de informação, comunicação e redes sociais, pelo crescimento exponencial da digitalização de redes e serviços e pela popularização de smartphones e serviços de banda larga, acabaram gerando um ambiente tecnológico transformador, que modificou e está modificando o mercado. Como notado por Reichheld e Scheflter (2000, p. 107), “word of mouse se espalha mais rápido que word of mouth".

Ferrel et al. (2015) ponderam que uma formação mais quantitativa em marketing, no futuro, parece ser uma tendência. De fato, O'Brien (2016) comenta que conhecer o big data, ter uma mentalidade abrangente do negócio, demonstrar impacto quantitativo e liderar de maneira proativa a cooperação dos C-Executives da organização estão entre os principais fatores de sucesso dos CMO. 
A maioria dos CMO, contudo, não teve formação aderente a todas essas necessidades. Nadeem (2015), por exemplo, ressalta que a utilização de dados já é uma realidade há algum tempo, mas ainda há uma desconfiança nos seus resultados causada por falta do conhecimento sobre o assunto. Smith (2018), por sua vez, aponta uma inabilidade latente na utilização de ferramentas preditivas e prescritivas baseadas em dados e algoritmos que permitem analisar e engajar os clientes individualmente.

Embora seja uma questão mundial, esse cenário de formação no Brasil se mostra mais crítico. Souza, Gehard e Brito (2018) observam que, se, nos Estados Unidos, o ensino de administração - e, por consequência, o de marketing - se caracteriza por um quadro de disciplinas mais flexível, com melhor balanço entre prática e teoria e mais interdisciplinaridade, aqui essa formação é mais rígida, teórica e menos interdisciplinar, o que torna os cursos e os profissionais de marketing menos preparados para o cenário em que hoje vivemos.

Não podemos deixar de considerar que a transformação digital também é cultural. Assim, muitos CMO não são, usando uma classificação proposta por Kaufman e Horton (2015), digital natives ou immigrants. Ou seja, a cultura digital não é sua cultura nativa ou adotada, o que dificulta seus insights e pode mitigar o impacto de sua experiência, que era um elemento importante para sua análise e decisão. Essa situação é particularmente grave, segundo Nath e Mahajan (2015, 2017), porque, quando acontece o distanciamento do CMO do resultado de vendas, há maior probabilidade do seu turnover.

\section{A PARCERIA ENTRE O CMO E O CIO: QUESTÃO DE INTELIGÊNCIA}

Em 2017, nos Estados Unidos, os CMOs investiram mais em tecnologia da informação (TI) do que seus pares CIOs (Sorofman, 2017), e esse desbalanceamento de autoridade tem criado certa rivalidade entre esses 2 profissionais acerca das decisões de orçamento de capital e de priorização de ações nessa área (Whitler et al., 2017). Assim, a gestão da relação CMO-ClO se torna vital à luz da magnitude dos investimentos envolvidos, cuja estimativa para 2021 é de 4.018 trilhões de dólares (Gartner, 2019) e pela crença de que, para a maioria dos CEOs americanos, tecnologia da informação é um fator crítico de sucesso para a performance corporativa (International Business Machines [IBM], 2010).

Nos últimos 25 anos, grandes tendências em tecnologia, como internet, computação em nuvem, dispositivos móveis, redes sociais e comercio eletrônico, transformaram os cenários de negócio de forma dramática, o que afeta inexoravelmente os papéis dos CMOs e dos CIOs, compelindo-os à cooperação (Taylor \& Vithayathil, 2018; Wiedeck \& Engelen, 2018). Assim, à medida que o uso de ferramentas digitais se intensifica, espera-se um adensamento da colaboração entre o CMO e o ClO (NealeMay, 2010). Entretanto, pesquisas mostram que, conforme essa relação se torna mais crítica, fica também mais problemática (Whitler et al., 2017) e se reflete na proclamação da Associação Americana dos Distribuidores de Energia (National Association of Electrical Distributors [NAED], 2014): "Marketing é de Marte, Tl é de Vênus". As tensões no relacionamento entre o ClO e o CMO se refletem em pesquisas que mostram que a maioria dos $\mathrm{CMOs}$ americanos se vê como líder e responsável natural pelos esforços de big data, enquanto a maior parte dos CIOs se percebe também nesse papel (Muse, 2013).

Para assegurar um equilíbrio entre funções e reponsabilidades do CMO e do CIO, Whitler et al. (2017) fazem algumas recomendações:

1. É fundamental que o CEO garanta simetria e equidade dentro da hierarquia da empresa, assegurando os mesmos status e nível de report para ambos os cargos.

2. O CEO deve estabelecer perspectivas e metas comuns para o $\mathrm{ClO}$ e o $\mathrm{CMO}$, além dos recursos necessários para seu atingimento, com as respectivas responsabilidades e metas individuais, desenhando uma estrutura colaborativa e sem redundâncias para ambos.

Além disso, observa-se que os CMOs não podem continuar focados somente em estratégia e criatividade; precisam também se desenvolver nas ciências analíticas para serem capazes de identificar e capturar oportunidades de negócios, o que implica uma fluência em dados, indicadores, e, sobretudo, uma capacidade de definir sua visão de negócios de forma precisa, de modo que seus pares em tecnologia da informação a entendam (Ariker, 2012; Sleep \& Hulland, 2019). 
A gestão ativa do relacionamento do $\mathrm{CMO}$ e do $\mathrm{CIO}$ por parte do $\mathrm{CEO}$ é fundamental para que ambos percebam que são parceiros naturais. CMOs estão de posse de uma quantidade de dados e consumidores sem precedente, de onde se podem extrair insights que derivem em aumento das receitas e dos lucros. Já o ClO tem a expertise no desenvolvimento das arquiteturas de $\mathrm{Tl}$ e experiência na execução de grandes projetos de implementação de sistemas necessários à criação da espinha dorsal de big data da empresa, que vai permitir a geração de ideias para o marketing (Ariker, Harrysson \& Perrey, 2014).

\section{A PARCERIA ENTRE O CMO E O CSO: QUESTÃO DE PERFORMANCE}

Nem todos os CMOs têm responsabilidade por vendas (Malter \& Ganesan, 2005), e nos últimos anos tem havido ciclos sucessivos de combinação e separação das atividades de vendas e marketing (Verhoef \& Leeflang, 2009). Argumenta-se que a integração de atividades e responsabilidades de marketing e vendas é fator de performance superior para as empresas (Carpenter, 1992; Court, 2007; Dewsnap \& Jobber, 2000; Donath, 2004; Rosenbloom \& Anderson, 1984; Rouziès et al., 2005). Existe, de fato, alguma evidência empírica de que uma forte cooperação entre áreas distintas de marketing e vendas pode proporcionar esse nível de eficácia (Guenzi \& Traiolo, 2007), mas a maior parte dos pesquisadores que advogam pela integração entre as duas áreas sugere que ter um líder em comum para as duas áreas é uma das formas de conseguir isso (Cespedes, 1996; Donath, 2004; Rouziès et al., 2005).

Há base para afirmar que o poder, o prestígio e a autoridade do CMO aumentam quando seu descritivo de cargo inclui responsabilidade compartilhada sobre vendas, o que acontece pelos seguintes motivos (Nath \& Mahajan, 2011):

1. A função de vendas é uma fonte rica e informal de dados sobre os consumidores, pois o pessoal de vendas tem a expertise e o relacionamento com segmentos de mercado que estão sob sua responsabilidade;

2. A responsabilidade adicional pelas vendas torna o $\mathrm{CMO}$ mais preciso na alocação de seus recursos. Deriva-se daí que, conforme as conclusões de Piercy (1989), outro fator de incremento no poder e no prestígio do CMO é o controle sobre a previsão de vendas;

3. Quando o CMO também é responsável pelas vendas, seu papel passa a ser central na C-Suite, em especial pelo aumento de oportunidades de integração com seus pares.

\section{A LINGUAGEM COMUM ENTRE O CMO, SEUS PARES E O CEO: QUESTÃO DE SOBREVIVÊNCIA}

Como notado por Court (2007), não é alta a quantidade de CEOs com experiência em marketing, sendo a formação mais predominante finanças e operações. Isso resulta, muitas vezes, em não entendimento do papel/perfil do CMO, do seu trabalho e dos conceitos que os norteiam, trazendo graves problemas de comunicação e de alinhamento entre eles, além de uma não valorização do trabalho dos seu CMO, como apontado por Whitler e Morgan (2007).

Nessas condições, o CEO pode não fornecer os recursos necessários para a realização eficiente do trabalho do CMO (Whitler \& Morgan, 2017) ou se envolver de modo insuficiente nas ações de marketing, não cumprindo seu papel de facilitar o alinhamento das ações das diversas áreas da empresa com a estratégia empresarial escolhida (Court, 2007). Também é possível que o CEO subestime os efeitos de longo prazo das ações de marketing, priorizando de ações de curto prazo, reconhecíveis com mais rapidez, mas que não necessariamente trarão valor para o acionista em horizonte mais longo (O’Brien, 2016).

Esse cenário pode se tornar mais crítico caso haja uma substituição do CEO. Nath e Mahajan (2017) demonstram que o turnover de CMO é maior durante a sucessão do CEO, em particular quando o novo profissional classifica os resultados da empresa como abaixo da expectativa.

Por outro lado, os CMOs podem se mostrar incapazes de explicar e demonstrar para o CEO e seus pares a importância de suas ações e/ou o alinhamento destas com os objetivos da organização. Podem apresentar também uma dificuldade de associar suas ações com os resultados empresariais (O'Brien, 2016). De fato, muitos profissionais de marketing têm 
dificuldades em quantificar, em resultados financeiros, termos como brand equity, customer equity, persuasion, loyalty, customer satisfaction e digital influencers, por exemplo, que são variáveis-chave no sucesso de qualquer negócio, mas que não têm um corolário fácil em finanças (Klaus et al., 2014).

As deficiências de formação dos CMO discutidas no tópico anterior dificultam o desenvolvimento do que O'Brien (2016) definiu como uma língua franca entre CMO e CEO, baseada em conceitos econômicos e financeiros, mas que pode ser acrescida de conceitos de marketing, de modo a criar um território comum e inteligível para ambas as partes.

Além disso, a relação interpessoal com o CEO é um fator crítico de sucesso para a estabilidade do CMO no cargo. Uma pesquisa da empresa de consultoria Booz \& Company (McGovern \& Quelch, 2004), de 2004, já apontava que uma das razões para um alto turnover desses profissionais de marketing é o desconforto gerado no CEO por alguém que o ofusca. Assim, o estudo recomenda que é vital um entendimento, por parte de ambos os profissionais, de que o CEO é o principal promotor da marca corporativa e que, na maioria dos casos, é seu rosto que aparece para a opinião pública e a imprensa - mas este precisa ser guiado e aconselhado por um especialista em marketing. $\mathrm{O}$ estudo ainda recomenda que, além da análise dos skills e da experiência do CMO na hora da contratação, é importante ter certeza de que, do ponto de vista de personalidade, os dois profissionais sejam compatíveis.

\section{O “SUPER-CMO": FRACIONAMENTO E TERCEIRIZAÇÕES}

Como luta pela sua legitimidade, credibilidade e autoridade em muitas organizações, segundo Nadeem (2015), o CMO ainda sofre uma erosão de suas funções originais com a criação novas funções C-level - como customer experience (CX), inovação e geração de demanda -, gerando uma disputa que acarreta subdivisões e sobreposições organizacionais. O CMO que lidera o crescimento do negócio e compartilha com seus pares a responsabilidade pela lucratividade da companhia com grande experiência e autoridade, que se destaca tanto em habilidades analíticas quanto criativas, tanto na ação tática quanto na estratégica, que tem domínio da cultura e das novas ferramentas digitais, que tem a confiança do CEO e o respeito de seus pares, esse CMO se torna um profissional com uma qualificação ímpar, quase um super-CMO - cada vez mais raro.

Wech (2017, p. 59) diz: “Hoje, os CEOs desejam um CMO que seja o líder capaz de gerar crescimento da companhia, mas nem todo executivo de marketing tem habilidades, capacidade, experiência e liderança para gerar receitas, lucratividade, e, simultaneamente, se adaptar a uma nova cultura digital".

Uma alternativa para mitigar essa situação é a criação do fractional CMO, ou CMO fracionado. Uma das primeiras vezes em que essa expressão apareceu foi na matéria da Forbes de 2015 (Whitler, 2015), "The Outsourced Executive: A Growing Leadership Staffing Solution", em que o fractional CMO, também definido como CMO terceirizado ou part time, é descrito como um profissional estratégico e experiente, que proporciona às empresas que o contratam a experiência de ter um super-CMO em tempo parcial. Segundo a mesma matéria, os CMOs fracionados são uma boa opção para empresas de tamanho médio ou startups que não precisam ou não podem pagar um executivo sênior de marketing permanente e full time, mas que enxergam enormes benefícios na contribuição de curto e médio prazos que um profissional experiente, já testado e aprovado no mercado, pode trazer. Além de ser parte temporariamente da C-Suite, o CMO fracionado também pode conduzir workshops na companhia, ser coach ou mentor de executivos menos experientes, ou mesmo conduzir auditorias de marketing.

Porém, se há demanda por um CMO pleno, com todas as suas funções originais, mas poucos profissionais são capacitados para ocupar essa vaga, há que explorar a questão da formação. Para poderem competir de forma bem-sucedida na era digital, alunos e profissionais de marketing devem dominar as ferramentas analíticas intrínsecas a esse ambiente, o que requer mudanças nas habilidades e nos conhecimentos desejados pelos empregadores (Schlee \& Karns, 2017). Como resultado, há uma demanda imperiosa para que profissionais de marketing desenvolvam e dominem as ferramentas necessárias para servir a um consumidor digital, sob pena de se tornarem obsoletos (Langan, Cowley \& Nguyen, 2019). Nesse contexto, uma pesquisa realizada com aproximadamente 1.000 profissionais de marketing de companhias americanas e inglesas mostrou que somente $8 \%$ estavam familiarizados com ferramentas de marketing digital básicas, e mais de dois terços admitia a necessidade de melhorar as habilidades na área para se manter competitivo profissionalmente (O'Brien, 2016). 
Os avanços recentes em tecnologia afetaram virtualmente todos os aspectos do marketing. Como dito antes, a combinação de right (creative) e left (analytical) brain skills requer um perfil específico e grande capacidade das instituições de ensino para desenvolvê-las em sua plenitude (Kerr \& Kelly, 2017). Assim, Ferrel et al. (2015, p. 172, tradução nossa) consideram que o "desafio dos professores é como transmitir o importante papel estratégico do marketing num curso tradicionalmente muito focado em tática" e que "estudantes mais orientados qualitativamente podem pensar que marketing seja fácil e simples". De forma mais assertiva, Rohm, Stefl e Saint Clair (2018) propuseram um programa de marketing fundamentado em disciplinas digitais como forma de acelerar o fechamento do gap da formação dos atuais profissionais de marketing.

Outras iniciativas nesse sentido estão sendo conduzidas por instituições que já diagnosticaram essa necessidade. Segundo matéria da Bloomberg Business Week (Zaleski \& Barinka, 2019), a Universidade da Califórnia-Berkeley inaugurou uma "graduação dual" em engenharia e administração, por meio de um programa acadêmico novo, a ser concluído em 4 anos, batizado de Management, Entrepreneuship \& Technology (MET). O objetivo do curso é a formação de profissionais multifacetados, fluentes tanto na língua dos negócios quanto nas novas tecnologias. Em 2019, o curso recebeu 2.500 inscrições, tendo admitido apenas $3 \%$ dos candidatos.

Todavia, até onde, as escolas de administração estão, de fato, oferecendo e enfatizando o desenvolvimento de habilidades analíticas e digitais? Uma pesquisa realizada por Langan et al. (2019) buscou descobrir como essas disciplinas estavam sendo incorporadas aos programas acadêmicos das universidades americanas. 0 exame foi realizado nas 529 instituições americanas filiadas à Association to Advance Collegiate Schools of Business (AACSB), com o objetivo de entender em que formatos a capacitação digital estava sendo oferecida aos alunos - disciplinas eletivas, obrigatórias e de especialização - e como estava sendo a aceitação destes. Além disso, foi o primeiro estudo a explorar o perfil do professor encarregado de ministrar essas mesmas disciplinas. Algumas das principais descobertas da pesquisa foram:

- Há uma tendência crescente de tornar as disciplinas de marketing digital - em especial aquelas com grande carga analítica - uma condição obrigatória para a formatura, além de uma oferta cada vez maior de cursos de especialização.

- Já há uma maioria de $72 \%$ das instituições filiadas à AACSB cujos departamentos de marketing oferecem pelo menos 1 curso de marketing digital.

Dada a proficiência necessária para lecionar disciplinas no universo digital e a grande rapidez com a qual esses assuntos evoluem, os autores da pesquisa assumiram que tais disciplinas seriam lecionadas por profissionais da área atuando como professores adjuntos. O resultado mostrou, entretanto, que professores assistentes - de formação $100 \%$ acadêmica respondem pela maioria das disciplinas digitais, com exceção de marketing analytics e mídias sociais, que ficam a cargo dos professores adjuntos.

\section{PERSPECTIVAS DE PESQUISAS E CONSIDERAÇÕES FINAIS}

Estamos vivendo uma era de imensas transformações na função de marketing, em grande parte pelo efeito das novas tecnologias digitais. Para ser bem-sucedido nesse novo ambiente, há que tomar alguns cuidados. Primeiro, esclarecer para a companhia - e para o próprio $\mathrm{CMO}$, em especial - o que se espera da função plena de um professional de marketing. $\mathrm{O}$ ritmo acelerado das transformações que estamos vivendo cria um horizonte de novos potenciais negócios e prioridades para esses profissionais de marketing, que deverão liderar esforços de implementação dessas novas iniciativas por toda a companhia. Essas novas atividades vão tornar esses $\mathrm{CMO}$ s capazes de modelar um novo perfil de atuação de suas companhias, incluindo a criação de vantagens competitivas fora do escopo original do departamento de marketing. Segundo, os próprios papéis do marketing e do CMO se expandem, e se tornará cada vez mais fundamental que os CEOs tenham a pessoa certa no cargo, a fim de que se tenha certeza de que inputs e insights vindos dos consumidores estão sendo transformados em novas oportunidades de negócio.

A despeito da alta volatilidade das atuais taxas de turnover dos CMOs, ainda não se pode afirmar que elas se manterão nesse patamar. É muito importante que o CMO e o CEO analisem, de forma racional, as expectativas em relação à função do CMO, cheguem a um acordo sobre o que define o sucesso esperado e, acima de tudo, estejam dispostos a se adaptar às rápidas mudanças desse novo mundo digital. 
Não se pode esquecer também que há um dever de casa a ser feito pelos atuais CMOs, no sentido de buscarem aperfeiçoamento e autodesenvolvimento para adquirir novas habilidades analíticas e digitais. Sem isso, não se pode falar em reinvenção da função. Para os novos CMOs em formação, há uma enorme responsabilidade das instituições de nível superior, no sentido de adaptarem e atualizarem seus programas para fazer frente às novas demandas que se apresentam, e ainda há muito a fazer nesse sentido.

Vários CMOs ainda têm um escopo de trabalho definido de forma muito estreita, com ênfase em publicidade, gestão de marcas e pesquisa de mercado. Muitos desses profissionais, sujeitos ao enorme turnover da profissão atualmente, são executivos bastante talentosos, o que torna esse processo particularmente frustrante para eles. Assim, para que se possam mitigar as chances desse turnover no futuro, recomenda-se que o $\mathrm{CMO}$ desenvolva bons relacionamentos com seus pares da C-Suite, em especial com o CEO. Com este, é fundamental o estabelecimento de metas ambiciosas, mas factíveis, para o marketing. Um CMO com o perfil certo, na função certa, pode ser um importante catalizador para o crescimento de uma empresa.

O marketing está hoje no exato ponto onde grandes forças, que moldam as mudanças nos atuais modelos de negócio, estão colidindo: estamos vendo um cenário turbulento, dúbio e incerto, pautado pela conectividade digital e móvel; a emergência do uso de big data e modelos estatísticos preditivos e prescritivos; o amplo e universal uso de mídias sociais, que desafiam o status quo e provocam mudanças econômicas e comportamentais.

Embora esse contexto seja desafiador para os $\mathrm{CMO}$, representantes do marketing na C-Suite, o ritmo acelerado das mudanças também cria um horizonte de novos potenciais negócios e prioridades para os $\mathrm{CMO}$ como líderes e facilitadores de esforços de implementação dessas novas iniciativas por toda a companhia. Essas novas atividades podem tornar os CMOs capazes de modelar um novo perfil de atuação de suas empresas e criar vantagens competitivas, além do escopo original do departamento de marketing. Ou seja, o CMO poderá ser o elemento-chave de liderança e coordenação das atividades de marketing na empresa e o catalizador da voz do cliente.

Para que o cenário de alto turnover se dissipe e a função de CMO ganhe mais perenidade dentro da empresa, os estudos parecem indicar a necessidade de nova abordagem da função, da formação do CMO e das inter-relações entre este e os outros executivos $C$-Suite. Por outro lado, ainda existem poucos estudos que mostrem quantitativamente as relações e a importância dos diversos aspectos abordados, como carência de formação digital e estatística; a necessidade de melhora das capacidades negociais e comunicação e do fortalecimento das relações interpessoais com o CEO e seus pares da C-Suite, além de mais trabalhos sobre as causas do turnover dos $\mathrm{CMO}$ diante desse novo cenário de mercado. Essas lacunas podem ser o objeto de futuras linhas de pesquisa que aprofundem relações causais da permanência e da relevância dos CMO nas organizações diante de tal contexto.

Longe de encerrar qualquer discussão a respeito, o objetivo deste ensaio foi contribuir para trazer à luz o debate sobre um fenômeno que encerra causas e efeitos bastante complexos, que demandam níveis distintos de análise por segmento e perfil de atuação das companhias e que estão submetidos a relações de causa e efeito nem sempre claras e identificáveis, tanto para o profissional quanto para a organização, de modo amplo aqui discutidos. 


\section{REFERÊNCIAS}

Alderson, W. (1957). Marketing behavior and executive education. Homewood, IL: Irwin.

Ariker, M. (2012, outubro 09). The one tool you need to make big data work: the pencil. Forbes Magazine. Recuperado de https://www. forbes.com/sites/mckinsey/2012/10/09/the-one-tool-you-need-tomake-big-data-work-the-pencil/?sh=55f8944b3a8a

Ariker, M., Harrysson, M., \& Perrey, J. (2014, agosto 01). Getting the $\mathrm{CMO}$ and $\mathrm{ClO}$ to work as partners. McKinsey \& Company. Recuperado de https://www.mckinsey.com/business-functions/mckinsey-digital/ our-insights/getting-the-cmo-and-cio-to-work-as-partners

Carpenter, P. (1992, março). Bridging the gap between marketing and sales (pp. 29-31). Sales \& Marketing Management.

Cespedes, F. V. (1996, Spring). Beyond teamwork: how the wise can synchronize. Marketing Management, 5(1), 24-37.

Court, D. (2007, agosto 01). The evolving role of the CMO. McKinsey Quarterly. Recuperado de https://www.mckinsey. com/business-functions/marketing-and-sales/our-insights/ the-evolving-role-of-the-cmo

Crosby, L. A., \& Johnson, S. L. (2005). Growing up. Marketing Management, 14(2), 12-13.

Dewsnap, B., \& Jobber, D. (2000, Spring). The sales-marketing interface in consumer packaged-goods companies: a conceptual framework. Journal of Personal Selling \& Sales Management, 20(2), 109-119.

Donath, B. (2004). 10 tips help align sales, marketing teams. Marketing News, 38(12), 5-7.

Fournaise Marketing Group. (2011). 73\% of CEOs think marketers lack business credibility: they can't prove they generate business growth. Recuperado de https://www.fournaisegroup.com/ marketers-lack-credibility/

Fuchs, C., \& Diamantopoulos, A. (2010). Evaluating the effectiveness of brand-positioning strategies from a consumer perspective. European Journal of Marketing, 44(11/12), 1763-1786.

Gartner. (2019, outubro 23). Gartner says global it spending to grow 3.7\% in 2020. Recuperado de https://www.gartner.com/en/newsroom/ press-releases/2019-10-23-gartner-says-global-it-spending-to-grow3point7-percent-in-2020

Gee, R. (2016, agosto 05). Undervalued or promiscuous? CMO turnover reaches its highest ever level. Marketing Week. Recuperado de https://www.marketingweek.com/undervalued-or-promiscuouscmo-turnover-reaches-its-highest-ever-level/

Germann, F., Ebbes, P., \& Grewal, R. (2015). The Chief Marketing Officer matters! Journal of Marketing, 79(3), 1-22.

Grönroos, C. (2006). On defining marketing: finding a new roadmap for marketing. Marketing Theory, 6(4), 395-417.

Guenzi, P., \& Traiolo, G. (2007, fevereiro). The joint contribution of marketing and sales to the creation of superior customer value. Journal of Business Research, 60(2), 98-107.
Gummesson, E., Kuusela, H., \& Närvänen, E. (2014). Reinventing marketing strategy by recasting supplier/customer roles. Journal of Service Management, 25(2), 228-24.

International Business Machines. (2010, maio). Capitalizing on complexity: Insights from the global chief executive officer study. Recuperado de https://www.ibm.com/downloads/cas/1VZV5X8J

Kaufman, I. W., \& Horton, C. (2015). Digital marketing: integrating strategy and tatics and values. New York, NY: Routdledge.

Kerin, R. A. (2005, outubro). marketing renaissance: opportunities and imperatives for improving marketing thought, practice, and infrastructure. Journal of Marketing, 69(4), 12-14.

Kerr, G., \& Kelly, L. (2017). IMC education and digital disruption. European Journal of Marketing, 51(3), 406-420.

Kitchenham, B. (2004). Procedures for performing systematic reviews (Joint Technical Report). Keele, UK: Department of Computer Science, Keele University.

Klaus, P., Edvardsson, B., \& Maklan, S. (2014). Editorial - Back where we belong: marketing as the organization's core strategy. Journal of Service Management, 25(2).

Kohli, A. K., \& Jaworski, B. J. (1990). Market orientation: the construct, research propositions, and managerial implications. Journal of Marketing, 54(2), 1-18.

Kotler, P. (2003, Winter). The Decline of Marketing: from four Ps to one $\mathrm{P}^{\prime}$ (pp. 38-40). Market Leader.

Langan, R., Cowley, S., \& Nguyen, C. (2019, fevereiro 17). The state of digital marketing in academia: an examination of marketing curriculum's response to digital disruption. Journal of Marketing Education, 41(1), 32-46.

Malter, A., \& Ganesan, S. (2005, junho). The decline and dispersion of marketing competence. MIT Sloan Management Review, 46(4), 35-43.

McGovern, G. J., \& Quelch, J. A. (2004, novembro 30). The fall and rise of the CMO (originally published by Booz \& Company). Strategy + Business. Recuperado de https://www.strategy-business.com/ article/04406?gko $=70 \mathrm{e} 12$

McGovern, G. J., Quelch, J. A., \& Crawford, B. (2004). Bringing customers into the boardroom. Harvard Business Review, 82(11), 70-80.

Morgan, N. A., Whitler, K. A., Feng, H., \& Chasi, S. (2019). Research in marketing strategy. Journal of the Academy of Marketing Science, 47(1), 4-29.

Muse, D. (2013, maio 29). What do CMOs and CIOs really think of each other? Cio.com. Recuperado de https://www.cio.com/article/2385444/ what-do-cmos-and-cios-really-think-of-each-other-.html

Nadeem, M. (2015). Chief Marketing "analytics or digital" Officer [cmo]: is the "big data" alone adequate for firm's customer retention \& return on investment? British Journal of Marketing Studies, 3(8), 17-33.

National Association of Electrical Distributors. (2014). The changing role of information technology and marketing. Recuperado de https:// www.naed.org/NAEDDocs/Resources/Business\%20Tools/Technology/ Executive-Guide-11The_Changing_Roles_of_IT_and_Marketing.pdf 
Nath, P., \& Bharadwaj N. (2020). Chief marketing officer presence and firm performance: Assessing conditions under which the presence of other c-level functional executives matters. Journal of the Academy of Marketing Science, 48(4), 670-694.

Nath, P., \& Mahajan, V. (2008). Chief Marketing Officers: a study of their presence in firms' top management teams. Journal of Marketing, 72(1), 65-81.

Nath, P., \& Mahajan, V. (2011). Marketing influence in the C-Suite: a study of Chief Marketing Officer power in firms' top management teams. Journal of Marketing, 75(1), 60-77.

Nath, P., \& Mahajan, V. (2017). Shedding light on the CMO revolving door: a study of the antecedents of Chief Marketing Officer turnover. Journal of the Academy of Marketing Science, 45, 93-118.

O'Brien, C. (2016, setembro 21). Missing the mark: the digital marketing skills gap in the USA, UK, \& Ireland. Digital Marketing Institute. Recuperado de https://digitalmarketinginstitute.com/en-us/the-insider/ missing-the-mark-the-digital-marketing-skills-gap-in-the-usa-uk-ireland

O’Brien, D., Veenstra, J., \& Murphy, T. (2018, janeiro 22). Redefining the CMO (Deloitte Review, issue 22). Deloitte. Recuperado de https://www2.deloitte.com/us/en/insights/deloitte-review/issue$22 /$ redefining-the-role-of-the-cmo-chief-marketing-officer.html

Petticrew, M., \& Roberts, H. (2008). Systematic reviews in the social sciences: a practical guide. Hoboken, Nova Jersey: John Wiley \& Sons.

Piercy, N. (1989, Spring). The power and politics of sales forecasting: uncertainty absorption and the power of the marketing department. Journal of the Academy of Marketing Science, 17, 109-120.

Press, G. (2013, julho 10). Digital marketing battlefield map: CMO vs. $\mathrm{CIO}$ and Gartner vs. Forrester. Forbes. Recuperado de https://www. forbes.com/sites/gilpress/2013/07/10/digital-marketing-battlefieldmap-cmo-vs-cio-and-gartner-vs-forrester/?sh=f6479f55f1c6

Reichheld, F., \& Schefter, P. (2000). E-Loyalty: your secret weapon on the web. Harvard Business Review, 78(4), 105-13.

Rohm, A. J., Stefl, M., \& Saint Clair, J. (2018). Time for a marketing curriculum overhaul: developing a digital-first approach. Journal of Marketing Education, 41(1), 47-59.

Rosenbloom, B., \& Anderson, R. E. (1984). The sales manager: tomorrow's super marketer. Business Horizons, 27(2), 50-56.

Rouziès, D., Andreson, E., Kohli, A. K., Michaels, R. E., Weitz, B. A., \& Zoltners, A. A. (2005). Sales and marketing integration: a proposed framework. Journal of Personal Selling \& Sales Management, 25(2), 113-122. Recuperado de https://www.jstor.org/stable/40471998

Schlee, R. P., \& Karns, G. L. (2017). Job requirements for marketing graduates: are there differences in the knowledge, skills, and personal attributes needed for different salary levels? Journal of Marketing Education, 39(2), 69-81.

Silver, S. (2013, maio 20). Bring on the Super-CMO. Strategy \& Business. Recuperado de https://www.strategy-business.com/article/21649

Sleep, S., \& Hulland, J. (2019). Is big data driving cooperation in the c-suite? The evolving relationship between the chief marketing officer and chief information officer. Journal of Strategic Marketing, 27(8), 666-678.
Sorofman, J., (2017, janeiro 09). CMO Role expands with rising marketing budgets. Forbes Magazine. Recuperado de https://www.forbes.com/sites/gartnergroup/2017/01/09/ cmo-role-expands-with-rising-marketing-budgets/?sh=224cdff547b1

Souza, L. L. F., Gehard, F., \& Brito, E. P. Z. (2018). Desafios na formação em Administração no Brasil. Administração: Ensino e Pesquisa, 19(1), 1-31.

SpencerStuart. (2019). CMO transitions: the shuffle continues an alarming number of companies across industries have undergone a change in the top marketing role once again this year. Recuperado de https://www.spencerstuart.com/research-and-insight/ cmo-transitions-the-shuffle-continues

Srinivasan, R., \& Ramani, N. (2019). With power comes responsibility: how powerful marketing departments can help prevent myopic management. Journal of Marketing, 83(3), 108-125.

Strandvik, T., Holmlund, M., \& Grönroos, C. (2014), The mental footprint of marketing in the boardroom. Journal of Service Management, 25(2), 241-252.

Taylor, J., \& Vithayathil, J. (2018). Who delivers the bigger bang for the buck: $\mathrm{CMO}$ or $\mathrm{CIO}$ ? The Journal of Strategic Information Systems, 27(3), 207-220.

Verhoef, P., \& Leeflang, P. (2009). Understanding the marketing department's influence within the firm, Journal of Marketing, 73(2), 14-37.

Verhoef, P., \& Leeflang, P., Reiner, J., Natter, M., Baker, W., Grinstein, A., ... Saunders, J. (2011). A cross-national investigation into the marketing department's influence within the firm: toward initial empirical generalizations. Journal of International Marketing, 19(3), 59-86.

Verhoef, P., Lemon, K., Parasuraman, A., Roggeven, A., Schlesinger, L. \& Tsiros, M. (2009). Customer experience: determinants, dynamics and management strategies. Journal of Retailing, 85(1), 31-41.

Webster, F. E., Jr. (2005). Back to the future: integrating marketing as tactics, strategy, and organizational culture. Journal of Marketing, 69(4), 4-6.

Webster, F. E., Jr., \& Lusch, R. F. (2013). Elevating marketing: marketing is dead! Long live marketing! Journal of the Academy of Marketing Science, 41(4), 1-11.

Weinzimmer, L. G., Bond, E. U., Houston, M. B., \& Nystrom, P.C. (2013). Relating marketing expertise on the top management team and strategic aggressiveness to financial performance. Journal of Strategic Marketing, 11(2), 133-59.

Welch, G. (2004, julho). CMO tenure: slowing down the revolving door. Recuperado de www.spencerstuart.com/research/articles/744/

Whitler, K. (2011, outubro 04). The "disposable" cmo: the downside of high cmo turnover. Forbes Magazine. Recuperado de https://www. forbes.com/sites/kimberlywhitler/2011/10/04/the-disposable-cmothe-downside-of-high-cmo-turnover/?sh=6f9918733520

Whitler, K. (2015, julho 28). The outsourced executive: a growing leadership staffing solution. Forbes Magazine. Recuperado de https:// www.forbes.com/sites/kimberlywhitler/2015/07/28/the-outsourcedc-level-executive-a-growing-leadership-trend/?sh=4093d35da107

Whitler, K., Boyd, D., \& Morgan, N. (2017). The criticality of the CMO-CIO alignment. Business Horizons, 60(3), 313-324. 
Whitler, K., \& Morgan, N. (2017). Why CMOs never last and what to do about it. Harvard Business Review, 95(4), 46-54.

Wiedeck, C., \& Engelen, A. (2018, julho). The copycat CMO: firms' imitative behavior as an explanation for CMO presence. Journal of the Academy of Marketing Science, 46(4), 632-651.

Wirtz, J., Tuzovic, S., \& Kuppelwieser, V. (2014). The role of marketing in today's enterprises. Journal of Service Management, 25(2), 171-194.
Wright, M., \& Russell, D. (2012). Some philosophical problems for service dominant logic in marketing. Australasian Marketing Journal, 20(3), 218-223.

Zaleski, O., \& Barinka, A. (2019, março 08). The fast track to being a CEO-Engineer: a dual undergraduate program in engineering and business is gaining traction with students. Bloomberg Business Week. Recuperado de https://www.bloomberg.com/news/ articles/2019-03-08/the-fast-track-to-being-a-ceo-engineer

Mel Girão

ORCID: https://orcid.org/0000-0002-2511-6240

Doutoranda pela Escola de Administração de Empresas de São Paulo da Fundação Getulio Vargas (FGV EAESP); Mestre em Administração pela Pontifícia Universidade Católica do Rio de Janeiro (PUC-Rio); Professora convidada da Fundação Getulio Vargas (FGV); CMO e Co-fundadora da Klug Technology. E-mail: mel.girao@fgvmail.br

Erik Fernandes

ORCID: https://orcid.org/0000-0002-1266-8345

Doutorando pela Escola de Administração de Empresas de São Paulo da Fundação Getulio Vargas (FGV EAESP); Mestre em Administração pela Fundação Dom Cabral (FDC); Professor convidado da Fundação Dom Cabral (FDC); Co-fundador da tugB Consultoria. E-mail: erikfernandes@tugb.com.br 